Monatsschrift f. Geburtshülfe u. Gynäkologie 1930;86:170-174

\title{
Heinrich v. Peham
}

\section{Ein Nachruf von 7. Amreich.}

Am Vormittag des 21. Juli traf in Wien die erste Nachricht ein, daß Hofrat v. Peham in den Morgenstunden des gleichen Tages einem Herzschlage erlegen sei. Wenige Stunden später brachten die Tages-blätter die Bestätigung dieser Unglücksbotschaft und machten die Hoffnung zunichte, daß das erste Gerücht auf einem Irrtum beruhe. Man wußte zwar, daß Hofrat v. Peham seit Beginn dieses Jahres an Herzbeschwerden litt, aber selbst seine nächste Umgebung ahnte nicht, daß die tückische Erkrankung den kaum 60jährigen so rasch dahin-raffen werde. Heinrich von Peham hat am 3. Mai 1871 in Wien das Licht der Welt erblickt. Er absolvierte in Wien das Schottengymnasium und wendete sich dann dem Sbudium der Medizin zu. Am 1. Juli 1895 wurde er zum Doktor der gesamten Heilkunde promoviert. Noch als Student war er Demonstrator am gerichtlich-medizinischen Institut des Prof. Eduard Hoffmann und arbeitete in gleicher Stellung am Institut für allgemeine und experimentelle Pathologie unter Richard Paltauf. --Nach seinem Doktorat war er ein halbes Jahr auf der internen Klinik Prof. Schròtters als Hospitant tätig und trat am 1. Okt. 1896 als Operationszögling an die chirurgische Universitätsklinik Prof. Alberts ein. I1/) Jahre verwendete er auf seine chirurgische Ausbildung und

Heinrich v. Peham.

171

erwarb hier die gründliche Vorbildung, die er für das Spezialfach der Medizin, dem er sich widmen wollte, für die Gynäkologie als notwendig erachtete.

Am 1. April 1898 trat Peham in das OperationszÖglingsinstitut Chrobaks ein. Schon nach neunmonatlicher Tätigkeit als Operations-zögling machte ihn Chrobak, auf seine Tüchtigkeit aufmerksam ge-worden, zum Assistenten (1. Jänner 1900). Am 13. Juli 1904 habilitierte 


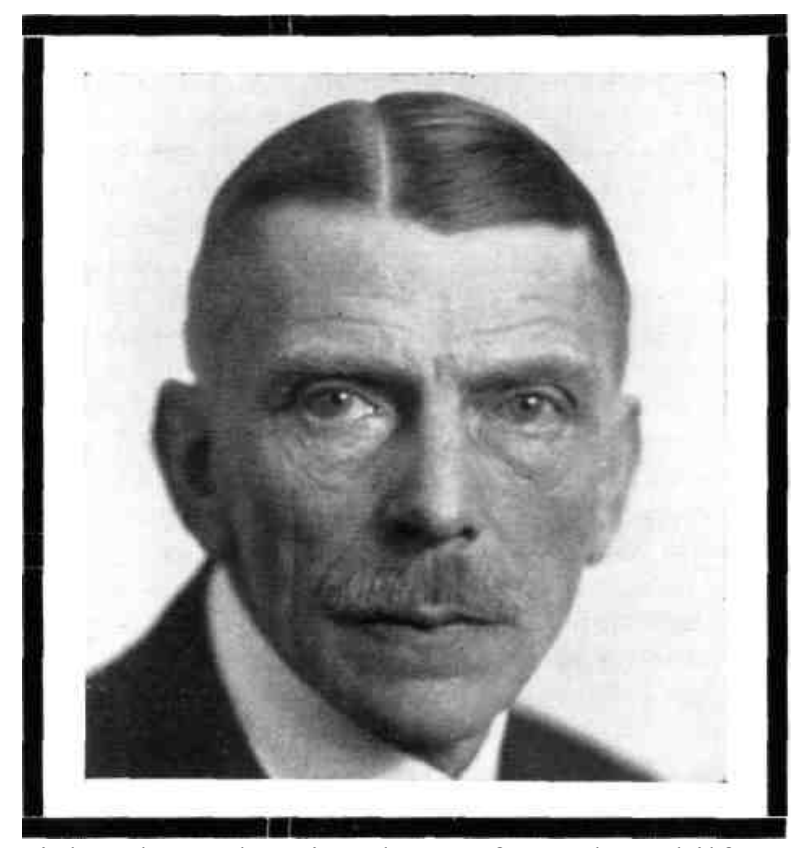

sich Peham als Privatdozent für Geburtshilfe und Gynäkologie an der Wiener Universität. Als sein Lehrer Chrobak am Schluß des Sommer-semesters 1907 auf Bitten und Drängen seiner Ereunde zur Schonung seiner angegriffenen Gesundheit von der Leitung der Klinik zurücktrat, supplierte Peham vom 1. Okt. 1907 bis 1. April 1908 die Lehrkanzel und war während dieser Zeit auch Examinator bei den medizinischen Prüfungen.

Die Jahre, die Peham an der Klinik Chrobaks verbrachte, dürften wohl zu den glücklichsten seines Lebens gehört haben. Chrobak schätzte ihn überaus hoch und förderte ihn auf jede nur mögliche Weise. Peham wieder hing mit größter Verehrung an seinem Lehrer. Bis an sein Ende, über alle Zeiten hinweg dauerte diese Liebe und Dankbarkeit gegen Chrobak. Als er längst selbst Chef der Schwesterklinik war, gedachte er oft und oft in rührender Anhänglichkeit seines alten Lehrers, rühmte seine liebevolle Menschlichkeit, seine bezaubernde Herzensgüte, seine stete Hilfsbereitschaft. Er stellte ihn uns Jungen hin als ein Muster eines offenen, ehrlichen Charakters und pries ihn als ein Vorbild treuer Pflichterfüllung.

Die innere Zufriedenheit mit seiner Stellung unter Chrobak drückt sich auch in einem ganz außerordentlichen Reichtum publizistischer

172

Heinrich. v. Fell am.

Tätigkeit aus. Aus dieser Zeit stammen nicht weniger als 21 Publi-kationen: Aus akzessorischen Nebennierenanlagen enfcstandene Ovarial-tumoren; eine Arbeit über Hyperplasie des Endometriums bei Myom; über zystische Myome des Uterus; über Karzinom der Bartholinschen Druse; über das traubige Sarkom der Cervix uteri; ferner die zusammen mit Keitler veröffentlichte Nachforschung über die Erfolge der kon-servativen Behandlung bei chronisch entzündlichen Adnextumoren; weiters eine Abhandlung über das primäre Karzinom der Tube; über einen Fall von Extrauteringravidität mit lebendem reifen Kind; über einen Fall von Verkümmerung der inneren Genitalien; über einen Fall von kongenitaler Atresie der Vagina; über einen Fall von Hyper -nephrom der linken Niere mit einer Metastase in der Vagina; über ein tuberkulöses Ulkus der Portio vaginalis. Außer diesen 12 gynäko-logischen Arbeiten stammen aus seiner Assistentenzeit nocli 9 Publi-kationen geburtshilflicher Natur: Ein Fall von Deciduoma malignum; ein Fall von Inversio uteri sub partu; ein Fall von Symphysen-ruptur; eine 
Arbeit über Pelvis inversa; über Uterusruptur in Narben; über einen Fall von Gravidität bei Uterus bicornis duplex; über die Serumbehandlung bei Puerperalfieber; über den Geburtsverlauf beim engen Becken; ferner sein Hauptwerk in dieser Zeit, die bei Alfred Holder erschienene Monographie über das enge Becken.

Als von Roslhorn am 1. April 1908 die Lehrkanzel seines Lehrers und Freundes Chrobak in Wien übernahm, schied Peham von der Klinik, an der er durch ein voiles Jahrzehnt tätig gewesen war. In der Folge hatte er durch $13 / 4$ Jahre kein klinisches Betätigungsfeld und widmete sich während dieser Zeit notgedrungen nur der Privatpraxis.

Im Dezember 1908 wurde Peham zum außerordentlichen Professor ernannt.

Ein Jahr später, im Dezember 1909, wurde er zum Leiter der gynäkologischen Abteilung der Wiener Allgemeinen Poliklinik bestellt und kam dadurch wieder zu einem größeren eigenen Krankenmaterial. Als gynäkologischer Primarius verblieb er 11 Jahre an der Poliklinik. Von den Arbeiten aus dieser Zeit seien nur die Abhandlung über Uterusmyome und deren Behandlung, über die Radiumbehandlung in der Gynäkologie und über die Röntgen- und Radiumbehandlung in der Gynäkologie hervorgehoben.

Am 22. Dez. 1920 erhielt Peham die Berufung als Leiter der I. Frauenklinik, die nach Schautas Tode zu Beginn des Jahres 1919 zuerst von Adler, dann von Thaler suppliert worden war. Trotzdem nun Peham durch seine große Privatpraxis, durch die Führung der Klinik, als Mitglied des Obersten Sanitätsrates, als wieder-holt gewählter Vorsitzender der Wiener Gesellschaft der Ärzte und der Wiener Gynäkologischen Gesellschaft in seiner Zeit außerordentlich beansprucht war, fand er doch noch auch in dieser letzten Periode seines Lebens Zeit zur Veröffentlichung zahlreicher wissenschaftlicher Arbeiten.

Von den geburtshilflichen Publikationen sei nur erinnert an: die Nachgeburtsperiode, die zirkuläre Abreißung der Cervix uteri sub partu, an die angeblichen Gefahren des Dämmerschlafes bei der Geburt, an die Schmerzverhütung und Schmerzstillung in der Geburtshilfe. Von dem nach dem Kriege besonders aktuell gewordenen Thema der ,,künstHeinrich v. Peham.

173

lichen Schwangerschaftsunierbrechung vom Standpunkte des Medi-ziners und des Juristen in Hinsicht auf die derzeit geltenden Gesetzes-bestimmungen" hat Peham den I. Teil, die Schwierigkeiten der ärzt-lichen Indikationsstellung zum künstlichen Abort, bearbeitet. Eine weitere wertvolle geburtshilfliche Arbeit betrifft die Bedeutung der Uterusnarben für Schwangerschaft und Geburt. In seiner gemeinsam mit Katz veröffentlichten Monographie über ,,die instrumentelle Perforation des graviden Uterus und ihre Verhütung" finden wir eine erschöpfende Darstellung dieses Themas mit Zugrundelegung einer sehr reichen Kasuistik. In den letzten Jahren war Peham hauptsächlich mit gynäkologischen Themen beschäftigt. Er hat das Kapitel ,,Myom” für das Stöckelsche Handbuch der Gynäkologie fertiggestellt und zusammen mit dem Verfasser dieses eine ausführliche gynäkologische Operationslehre herausgegeben, welche im Herbst dieses Jahres er-scheinen wird.

Außer diesen zahlreichen Publikationen wären noch zu erwähnen die Referate, Diskussionen und Demonstrationen in der Wiener gynäkologischen Gesellschaft, in der Gesellschaft der Ärzte in Wien und in der Krebsgesellschaft.

Als Wissenschaftler war Peham ein Feind jeder nichtssagenden Vielschreiberei. Für medizinisch-philosophische Grübeleien hatte er nicht viel übrig; nur Arbeiten, die Neues und nach menschlichem Erachten Richtiges brachten, schätzte er. Auch das überflüssige Reden in den wissenschaftlichen Gesellschaften haßte er. Ergriff er aber einmal das Wort, so konnte man 
sicher sein, daß hierzu ein wirklicher Grund vorlag, und daß er mit seinen Ausführungen den Nagel auf den Kopf traf.

Peham war ein vortrefflicher Organisator. Er hatte den Betrieb an der Klinik in so ausgezeichneter Weise eingerichtet, daß er nicht nur stets über alle Vorkommenheiten daselbst vollkommen orientiert war, sondern auch tatsächlich alles an der Klinik nach seinen Intentionen ausgeführt wurde. Auch Unzulänglichkeiten im Bau der Klinik wurden von ihm sofort erkannt und verbessert. War Peham Vorsitzender einer wissenschaftlichen Gesellschaft, so brauchte man nicht zu fürchten, daß die Diskussionen in einer einschläfernden Redeplätscherei verebbten, sondern konnte sicher sein, daß unter seiner geschickten Führung die Debatten zwar kurz, aber dafür zahlreich, sachlich, und das in Frage stehende Thema erschöpfend, sich aneinanderreihten. Als klinischer Lehrer verfügte er über eine glänzende Rednergabe. Alles in seinen Vorträgen fand seine logische Entwicklung, seine Sprech-weise war einfach, klar, präzis, für den Praktiker Nebensächliches wurde fortgelassen, stets das Wichtige des abzuhandelnden Gegenstandes von den verschiedensten Seiten beleuchtet und so den Hörern unvergeßlich eingeprägt. Besonders ausführlich wurden die Arten der Diagnosen-stellung und der Behandlung erörtert, die sich auch in der allgemeinen Praxis durchführen lassen, und nur in selteneren Fallen wurde gezeigt, was darüber hinaus die klinische Geburtshilfe und Gynäkologie zu leisten imstande ist. Me vergaß er den Menschen über der Wissen-schaft. Er nahm beim Unterricht auf die Patienten in jeder möglichen Weise Rücksicht und suchte sie durch eigenes Beispiel, dureb Belehrung der Studenten und durch klinische Verordnungen vor den Gefahren,

174 Heinrich v. Peham. - Personalien.

die in ihrer Benützung als Lehrmaterial liegen, so viel als möglich zu schützen. Aus alien diesen Gründen erfreute sich Peham der größten Beliebtheit bei den Studierenden. Sein Hörsaal war nicht nur stets bis zum letzten Platz besetzt, sondern seine Schüler saßen auch noch ãuf den Stiegen und Gängen, die zu den amphitheatralisch aufgebauten Bänken führten.

Als Operateur zeigte Peham jene große Ruhe, die nur solche Chirurgen besitzen, die die Technik der Operation und sich selbst vollkommen beherrschen. Dabei operierte er im Vollbewußtsein seiner Verantwortlichkeit für das Leben der Patientin mit der allergrößten Bedachtsamkeit und Gewissenhaftigkeit. Besonders vorsichtig war er in der Auswahl der zu operierenden Fälle und ging nur die wirklich aussichtsreichen an. Hierbei kam ihm seine hervorragende diagnostische Fähigkeit besonders zugute. Er vermochte nicht nur die feinsten Kleinig-keiten bei der Untersuchung zu tasten, sondern konnte auch die er-hobenen Befunde infolge seiner reichen Erfahrung und logischen Denkungsart richtig deuten.

Wegen seiner hervorragenden diagnostischen und operativen Fähig-keiten war Peham auch einer der gesuchtesten Ärzte Wiens. Seine Ruhe, sein menschliches Mitgefühl, seine große Erfahrung und seine strenge Gewissenhaftigkeit trugen noch das Weitere zu dem großen Erfolge bei, den er als Arzt und Operateur errungen hat.

Peham war ein Pflichtmensch wie sein Lehrer Chrobak, der das Vorbild seines Lebens war. Mit Hintansetzung seiner eigenen Inter -essen hat er vertreten, was sein Pflichtgefühl ihm vorschrieb, und manche Gegnerschaft ist ihm daraus erwachsen. Er war kerne Kämpfer-natur, seine ,, eiserne Maske" sollte nur sein warmfühlendes, leicht verwundbares Herz gegen die Menschen schützen. $\mathrm{Da}$ einem Manne von so hervorragenden Eigenschaften und in so hoher Stellung vielfache Auszeichnungen und Ehrungen zuteil wurden, ist begreiflich. Die Regierung des ehemaligen Kaiserreiches hat ihm den Orden der eisernen Krone III. Klasse u. a. verliehen. Er besaß zahlreiche ausländische Orden. Während des Weltkrieges wurde er in den Adelsstand erhoben und erhielt den Beinamen Ritter von Bojernberg. Im Jahre 1925 war er Vorsitzender der 
deutschen Gesellschaft für Gynäkologie. Für das Studienjahr 1927/28 wurde Peham durch das Vertrauen seiner Kollegen zum Rektor der Wiener Universität gewählt und erlangte damit die höchste erreichbare aka-demische Würde. Wiederholt war er Vorsitzender der Wiener gynäkologischen Gesellschaft und der Wiener Gesellschaft der Ärzte usw. Infolge seines mehr scheuen Wesens hielt sich Peham abseits dem lauten Getriebe der Welt. Er liebte über alles die Natur und ihre Pracht, die über Wald und Flur, über Berg und Tal verstreut liegt. Dort, in Hinterstoder, inmitten seiner bewunderten Berge, hat ihn ein rascher Tod ereilt.

Personalien.

Privatdozent Dr. Gustav Haselhorst in Hamburg erhielt die Amts-bezeichnung Professor. Dr. Werner Liittge in Erlangen hat sich für Geburtshilfe, Gynäko-logis und Röntgeriologie habilitiert. 